\title{
Results from $B$ factories in charmless (quasi)-two-body decays and Belle II prospects
}

\author{
Chia-Ling Hsu*十 \\ University of Melbourne \\ E-mail: chialinghestudent.unimelb.edu.au
}

In this proceeding, we report the recent studies in charmless two-body decays from $B$ factories and Belle II prospects on direct $C P$ violation in charmless $B$ decays. We present the first observation of the decay $B_{s}^{0} \rightarrow K^{0} \bar{K}^{0}$ using a dataset of $121.4 \mathrm{fb}^{-1}$ collected at the $\Upsilon(5 S)$ resonance with the Belle detector. We also discuss the first evidence of $B^{0} \rightarrow \eta \eta$. Being also related to direct $C P$ asymmetry, we present a preliminary measurement of the branching fraction and $C P$ asymmetry of $B^{+} \rightarrow K^{+} K^{-} \pi^{+}$decays. Furthermore, we discuss the future prospects on the studies of direct $C P$ violation in charmless $B$ decays at the coming Belle II experiment.

9th International Workshop on the CKM Unitarity Triangle

28 November - 3 December 2016

Tata Institute for Fundamental Research (TIFR), Mumbai, India

* Speaker.

${ }^{\dagger}$ for the Belle Collaboration 


\section{Introduction}

Charmless decays of $B$ mesons to three charged hadrons are suppressed in the Standard Model (SM), and thus provide an opportunity to search for physics beyond the SM through branching fraction enhancements. Large $C P$ asymmetries can also occur in these decays, due to interference of tree and loop diagrams with similar amplitudes; also there is the possibility of beyond-SM particles contributing in the loop diagrams. We present the resent results of charmless hadronic (quasi-)two-body $B_{s}^{0}$ and $B$ decays from the $B$ factories.

\section{The decay $B_{s}^{0} \rightarrow K^{0} \bar{K}^{0}$}

The two-body decays $B_{s}^{0} \rightarrow h^{+} h^{\prime-}$, where $h^{(1)}$ is either a pion or kaon, have now all been observed [8]. In contrast, the decays $B_{s}^{0} \rightarrow h^{0} h^{\prime 0}$ with neutral daughters have not been observed yet. The decay $B_{s}^{0} \rightarrow K^{0} \bar{K}^{0}$ [1] is predicted to have a relatively large branching fraction. In the $\mathrm{SM}$, the decay is dominated by $b \rightarrow s$ loop (or "penguin") transition as shown in Fig. 1, and the predicted branching fraction is in the range $(16-27) \times 10^{-6}$ [2]. The contribution due to non-SM particles or couplings could enhance the branching fraction [5]. It has been pointed out that $C P$ asymmetries in $B_{s}^{0} \rightarrow K^{0} \bar{K}^{0}$ decays are promising probe in which to search for new physics [3].

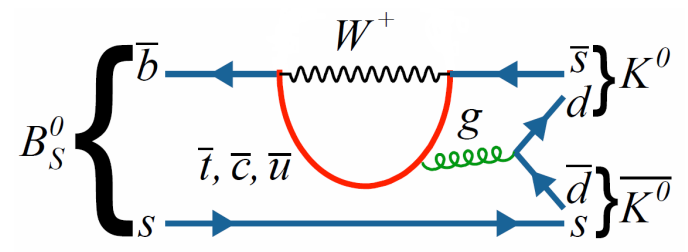

Figure 1: Loop diagram for $B_{s}^{0} \rightarrow K^{0} \bar{K}^{0}$ decays.

The previous upper limit on the branching fraction of $B_{s}^{0} \rightarrow K^{0} \bar{K}^{0}$ was set to be $6.6 \times 10^{-5}$ at $90 \%$ confidence level by the Belle Collaboration using $23.6 \mathrm{fb}^{-1}$ of data collected at the $\Upsilon(5 S)$ resonance [4]. Here, we present the updated result using the full data set of $121.4 \mathrm{fb}^{-1}$, which corresponds to $(6.53 \pm 0.66) \times 10^{6} B_{s}^{0} \bar{B}_{s}^{0}$ pairs [7], collected at the $\Upsilon(5 S)$. This analysis also uses improved tracking, $K^{0}$ reconstruction, and continuum $e^{+} e^{-} \rightarrow q \bar{q}(q=u, d, s, c)$ background suppression algorithms.

$K^{0}$ candidates are reconstructed from $K_{S}^{0} \rightarrow \pi^{+} \pi^{-}$decays with the invariant mass within 12 $\mathrm{MeV} / \mathrm{c}^{2}$ of the nominal $K_{S}^{0}$ mass [8]. Signal candidates are identified using two kinematic variables in the center-of-mass frame: the beam constrained mass, $M_{\mathrm{bc}} \equiv \sqrt{E_{\text {beam }}^{2}-p_{B}^{2}}$, and the energy difference, $\Delta E \equiv E_{B}-E_{\text {beam }}$, where $E_{\text {beam }}$ is the beam energy, and $E_{B}$ and $p_{B}$ are the reconstructed energy and momentum of $B_{s}^{0}$ candidates. To suppress the dominant background from the continuum process $e^{+} e^{-} \rightarrow q \bar{q}(q=u, d, s, c)$, a multivariate analyzer based on a neural network is employed. To discriminate the continuum background from signal, the variables describing the event topology are used in the neural network. The output of the neural network is transformed to

$$
C_{\mathrm{NN}}^{\prime}=\ln \left(\frac{C_{\mathrm{NN}}-C_{\mathrm{NN}}^{\min }}{C_{\mathrm{NN}}^{\max }-C_{\mathrm{NN}}}\right)
$$


where $C_{\mathrm{NN}}^{\min }$ and $C_{\mathrm{NN}}^{\max }$ are the minimum and the maximum variables of the neural network outputs, respectively. The distribution of $C_{\mathrm{NN}}^{\prime}$ can be well modeled by a Gaussian function, and can be used in the signal extraction later.

A three dimensional unbinned maximum likelihood fit to the variable $M_{\mathrm{bc}}, \Delta E$, and $C_{\mathrm{NN}}^{\prime}$ is performed to extract the signal yield. We obtain $29.0_{-7.6}^{+8.5}$ signal events and $1095.0_{-33.4}^{+33.9}$ continuum background events in the fit to data. Figure 2 shows the projections of the fit in the signal-enhanced region. The branching fraction of the decay $B_{s}^{0} \rightarrow K^{0} \bar{K}^{0}$ is:[9]

$$
\mathscr{B}\left(B_{s}^{0} \rightarrow K^{0} \bar{K}^{0}\right)=\left(19.6_{-5.1}^{+5.8} \pm 1.0 \pm 2.0\right) \times 10^{-6},
$$

where the first uncertainty is statistical, the second is systematic, and the third reflects the uncertainty due to the total number of $B_{s}^{0} \bar{B}_{s}^{0}$ pairs. This measurement is the first observation of this decay, and is in good agreement with the SM predictions [2].
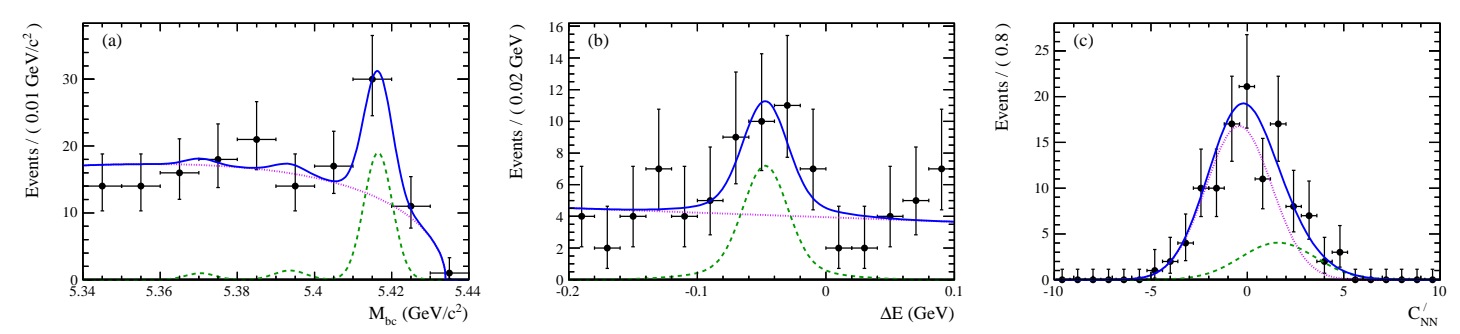

Figure 2: Projections of the 3D fit to the real data: (a) $M_{\mathrm{bc}}$ in $-0.11 \mathrm{GeV}<\Delta E<0.02 \mathrm{GeV}$ and $C_{\mathrm{NN}}^{\prime}>0.5$; (b) $\Delta E$ in $5.405 \mathrm{GeV} / c^{2}<M_{\mathrm{bc}}<5.427 \mathrm{GeV} / c^{2}$ and $C_{\mathrm{NN}}^{\prime}>0.5$; and (c) $C_{\mathrm{NN}}^{\prime}$ in $5.405 \mathrm{GeV} / c^{2}<M_{\mathrm{bc}}<$ $5.427 \mathrm{GeV} / c^{2}$ and $-0.11 \mathrm{GeV}<\Delta E<0.02 \mathrm{GeV}$. The points with error bars are data, the (green) dashed curves show the signal, (magenta) dotted curves show the continuum background, and (blue) solid curves show the total. The three peaks in $M_{\mathrm{bc}}$ arise from $\Upsilon(5 S) \rightarrow B_{s}^{0} \bar{B}_{s}^{0}, B_{s}^{* 0} \bar{B}_{s}^{0}+B_{s}^{0} \bar{B}_{s}^{* 0}$, and $B_{s}^{* 0} \bar{B}_{s}^{* 0}$ decays.

\section{Evidence of the decay $B^{0} \rightarrow \eta \eta$}

The $B^{0} \rightarrow \eta \eta$ decay is dominated by the $b \rightarrow u$ Cabibbo- and color-suppressed tree diagram and the $b \rightarrow d$ penguin diagram shown in Fig. 3. The branching fraction of this decay is expected to be $(0.3-3.1) \times 10^{-6}$, estimated from the calculations based on QCD factorization [10], soft collinear effective theory [11], SU(3) flavor symmetry [12] and flavor U(3) symmetry [13]. This decay plays an important role in improving the flavor $S U(3)$ calculations of $\left|S_{c c s}-S_{f}\right|$, where the final state $f$ is $\eta^{\prime} K$ or $\phi K$, the $C P$-violating parameter $S_{f} \sim \sin 2 \phi_{1}$ is measured in the time-dependent analysis [14], and the $C P$-violating parameter $S_{c \overline{c s}}$ is measured in the CabibboKobayashi-Maskawa(CKM)-favored $b \rightarrow c \bar{c} s$ decays. The bound on $\sin 2 \phi_{1}$ can be improved by the precise measurement of the branching fraction of $B^{0} \rightarrow \eta \eta[15,16]$.

This mode has been studied previously by Belle and BABAR [17, 18]. The best upper limit on this branching fraction is set to be $\mathscr{B}\left(B^{0} \rightarrow \eta \eta\right)<1.0 \times 10^{-6}$ at $90 \%$ confidence level (CL) by BaBar [18]. We update the previous Belle result by using the full data set of the Belle experiment running on the $\Upsilon(4 S)$ resonance at the KEKB asymmetric-energy $e^{+} e^{-}$collider [19]. This data set corresponds to $753 \times 10^{6} B \bar{B}$ pairs, which is a factor of five larger than in the previous Belle study. 


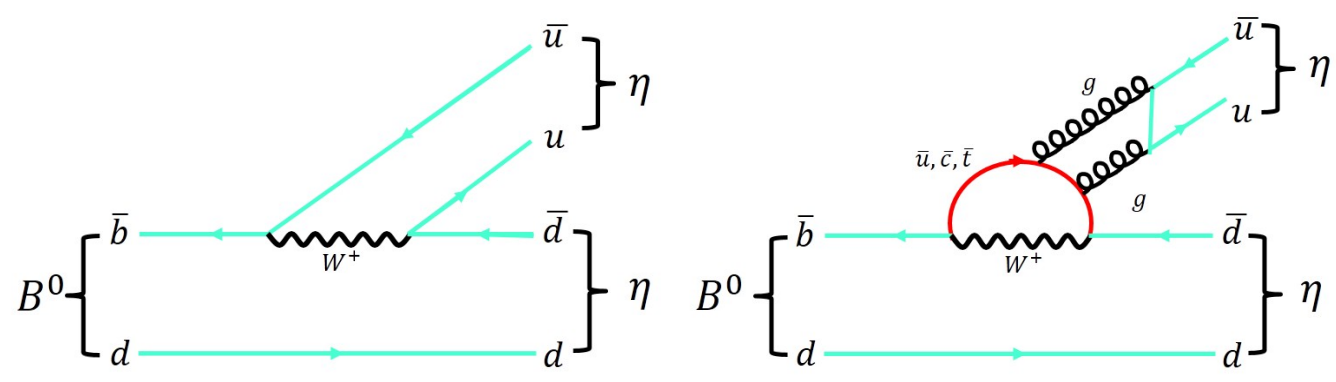

Figure 3: Diagrams for $B^{0} \rightarrow \eta \eta$ decays

The $\eta$ candidates are reconstructed from the sub-decay channels: $\eta \rightarrow \gamma \gamma\left(\eta_{\gamma \gamma}\right)$ and $\eta \rightarrow$ $\pi^{+} \pi^{-} \pi^{0}\left(\eta_{3 \pi}\right)$. A $\pi^{0}$ candidate is required to have a $\gamma \gamma$ invariant mass between 117 and $155 \mathrm{MeV} / c^{2}$, which corresponds to $\pm 3 \sigma$ around the nominal $\pi^{0}$ mass [8]. The $\eta_{3 \pi}$ candidates are reconstructed by two oppositely charged pions and a $\pi^{0}$ candidate. The invariant mass of $\eta_{\gamma \gamma}$ candidates is required to be in the range $476-579 \mathrm{MeV} / \mathrm{c}^{2}$, which corresponds to $\pm 2.5 \sigma$ around the nominal $\eta$ mass [8]; that of $\eta_{3 \pi}$ candidates is within the range $527-568 \mathrm{MeV} / c^{2}$ with $\pm 3 \sigma$ around the nominal mass. The $B^{0}$ candidates are identified using two kinematic variables in the center-of-mass frame: the beam constrained mass $M_{\mathrm{bc}} \equiv \sqrt{E_{\text {beam }}^{2}-p_{B}^{2}}$ and the energy difference $\Delta E \equiv E_{B}-E_{\text {beam }}$, where $E_{\text {beam }}$ is the beam energy, and $E_{B}$ and $p_{B}$ are the reconstructed energy and momentum of $B^{0}$ candidates. The $B^{0}$ candidates are required to satisfy $-0.3 \mathrm{GeV}<\Delta E<0.2 \mathrm{GeV}$ and $M_{\mathrm{bc}}>$ $5.25 \mathrm{GeV} / c^{2}$. The dominant background is from the continuum process $e^{+} e^{-} \rightarrow q \bar{q}(q=u, d, s, c)$, which is suppressed with a neural network. We transforme the neural network output $C_{\mathrm{NN}}$ to $C_{\mathrm{NN}}^{\prime}=\ln \left(\frac{C_{\mathrm{NN}}-C_{\mathrm{NN}}^{\min }}{C_{\mathrm{NN}}^{\max }-C_{\mathrm{NN}}}\right)$, where $C_{\mathrm{NN}}^{\min }$ is -0.8 and $C_{\mathrm{NN}}^{\max }$ is the maximum variables of the neural network outputs. This variable is also included in the signal extraction method.

Table 1: Summary of results of the combined fit

\begin{tabular}{cccc}
\hline \hline Sub-decay mode & $\eta_{\gamma \gamma} \eta_{\gamma \gamma}$ & $\eta_{\gamma \gamma} \eta_{3 \pi}$ & $\eta_{3 \pi} \eta_{3 \pi}$ \\
\hline Yields & & & \\
Signal & $23.6_{-6.9}^{+8.1}$ & $9.2_{-2.7}^{+3.2}$ & $2.7_{-0.8}^{+0.9}$ \\
Continuum & $3860.5_{-62.4}^{+63.1}$ & $3779.7_{-61.5}^{+62.0}$ & $621.4_{-24.8}^{+25.4}$ \\
$b \rightarrow c$ (fixed) & 5.9 & 5.9 & 2.2 \\
$b \rightarrow u, d, s$ (fixed) & 27.4 & 17.8 & 4.5 \\
$B^{0} \rightarrow \eta \pi^{0}$ (fixed) & 1.4 & 0.1 & - \\
Efficiency & & & \\
$\varepsilon_{\text {rec }}(\%)$ & 26.3 & 17.8 & 8.9 \\
$\prod \mathscr{B}_{\eta}(\%)$ & 15.5 & 8.9 & 5.1 \\
\hline \hline
\end{tabular}

The branching fraction of $B^{0} \rightarrow \eta \eta$ is obtained by a simultaneous fit to the $\eta_{\gamma \gamma} \eta_{\gamma \gamma}, \eta_{\gamma \gamma} \eta_{3 \pi}$ and $\eta_{3 \pi} \eta_{3 \pi}$ decay channels. We perform a three dimensional extended unbinned maximum likelihood fit to the variables $M_{\mathrm{bc}}, \Delta E$ and $C_{\mathrm{NN}}^{\prime}$. The results of the fit are summarized in Table 1, and the 

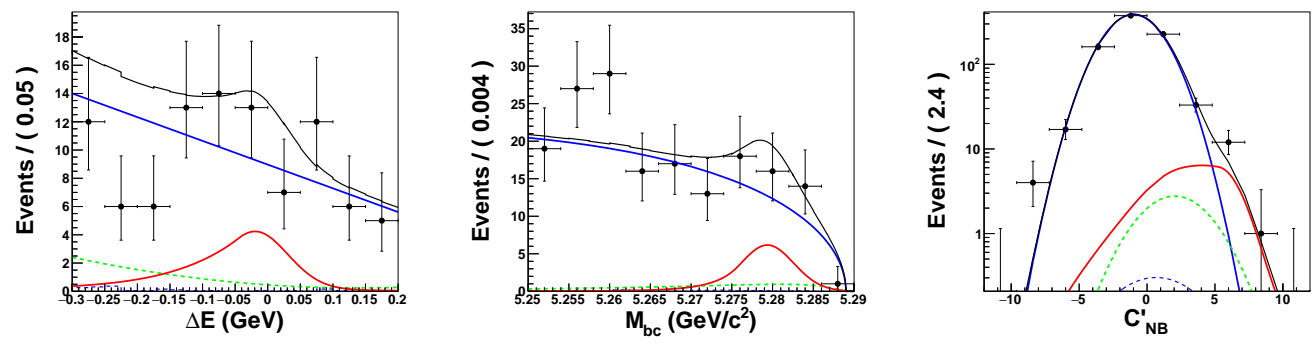

(a) $B^{0} \rightarrow \eta_{\gamma \gamma} \eta_{\gamma \gamma}$
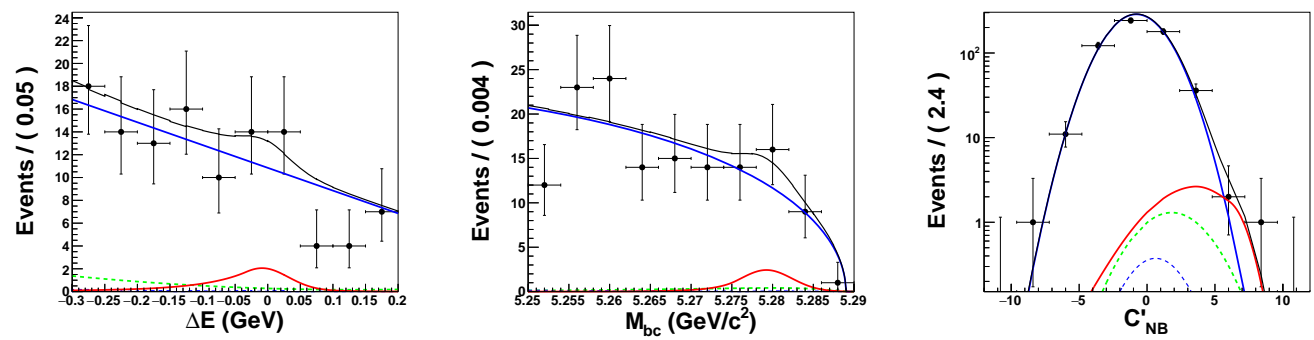

(b) $B^{0} \rightarrow \eta_{\gamma \gamma} \eta_{3 \pi}$
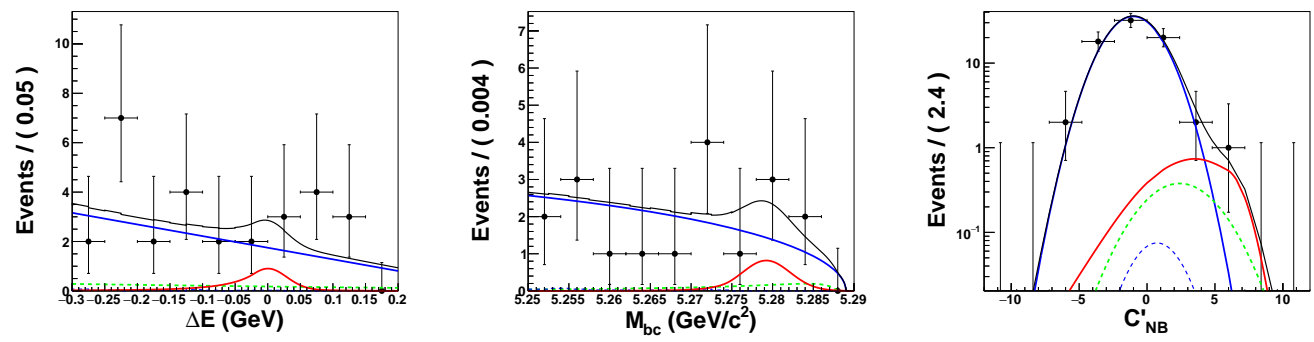

(c) $B^{0} \rightarrow \eta_{3 \pi} \eta_{3 \pi}$

Figure 4: Signal-enhanced projections of the simultaneous fit: The points with the error bars are the real data, the black solid line is total PDF, the red solid line show the signal, the blue solid line represent the $q \bar{q}$, the green dashed line is $b \rightarrow u, d, s$, and the blue dashed line is $b \rightarrow c$ background.

projections are shown in Fig. 4. The measured branching fraction is:[20]

$$
\mathscr{B}\left(B^{0} \rightarrow \eta \eta\right)=\left(7.6_{-2.3-1.6}^{+2.7+1.4}\right) \times 10^{-7},
$$

where the first uncertainty is statistical and the second is systematic. The significance of the result is $3.3 \sigma$ above zero, and provides the first evidence of this decay.

\section{Measurements of branching fraction and $C P$ asymmetry on $B^{+} \rightarrow K^{+} K^{-} \pi^{+}$}

The $B^{+} \rightarrow K^{+} K^{-} \pi^{+}$decay is dominated by the Cabbibo-suppressed $b \rightarrow u$ tree transition and $b \rightarrow d$ penguin shown in Figure 5. In recent years, unidentified structure has been measured by BaBar [21] and LHCb [22, 23] in the $K^{+} K^{-}$low-invariant-mass spectrum of the $B^{+} \rightarrow K^{+} K^{-} \pi^{+}$ decay. The LHCb studies revealed a non-zero inclusive $C P$ asymmetry of $-0.123 \pm 0.017 \pm$ $0.012 \pm 0.007$ and a large unquantified local $C P$ asymmetry in the same mass region. These results suggest that final-state interactions may be a contributing factor to $C P$ violation [24, 25]. We 
studied this decay using the full data set of the Belle experiment running on the $\Upsilon(4 S)$ resonance at the KEKB asymmetric-energy $e^{+} e^{-}$collider [19]. This data set corresponds to $772 \times 10^{6} B \bar{B}$ pairs.

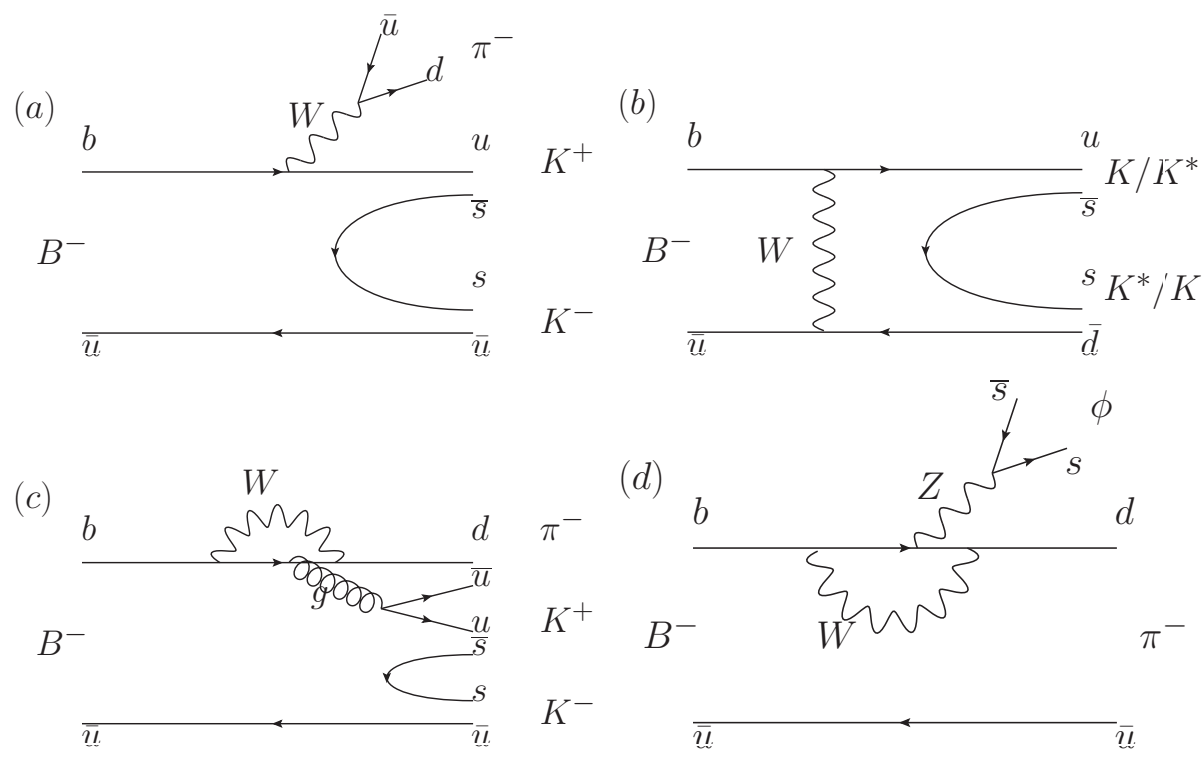

Figure 5: $B^{+} \rightarrow K^{+} K^{-} \pi^{+}$Feynman diagrams (all Cabibbo-suppressed). (a) tree diagram, (b) $W$-exchange diagram leading to $K K^{*}$ states, (c) strong penguin diagram, and (d) electroweak penguin leading to the $\phi \pi$ state.

To reconstruct $B^{+} \rightarrow K^{+} K^{-} \pi^{+}$, we combine two oppositely-charged kaons with a charged pion. Candidate $B$ mesons are also identified using two kinematic variables in the c.m. frame: the beam-energy constrained mass and the energy difference. The fit region is defined as $M_{\mathrm{bc}}>$ $5.24 \mathrm{GeV} / c^{2}$ and $|\Delta E|<0.3 \mathrm{GeV}$, while the signal-enhanced region is defined as $5.27<M_{\mathrm{bc}}<$ $5.29 \mathrm{GeV} / c^{2}$ and $|\Delta E|<0.05 \mathrm{GeV}$.

The dominant background is from continuum $e^{+} e^{-} \rightarrow q \bar{q}(q=u, d, s, c)$ processes. A neural network is used to suppress the continuum background. The neural network output $\left(C_{\mathrm{NN}}\right)$ selection requirement is optimized by maximizing a figure of merit defined as $N_{\mathrm{S}} / \sqrt{N_{\mathrm{S}}+N_{\mathrm{B}}}$ in the signalenhanced region, where $N_{\mathrm{S}}$ denotes the expected number of signal events based on MC simulation for a branching fraction of $5 \times 10^{-6}$, and $N_{\mathrm{B}}$ denotes the expected number of background events. We require the candidates with $C_{N N}>0.88$. Background contributions from $B$ decays via the dominant $b \rightarrow c$ transition (generic $B$ decays) are investigated with a MC sample of such decays. To suppress these backgrounds, the candidates for which the invariant mass of the $K^{+} K^{-}$or $K^{+} \pi^{-}$ system lies in range of $1850-1880 \mathrm{MeV} / \mathrm{c}^{2}$ are removed. The selection window corresponds to $\pm 3.75 \sigma$ around the nominal $D^{0}$ mass[8]. The charmless $B$ decays via $b \rightarrow q(q=u, d, s)$ processes are studied with a large MC sample where one of the $B$ mesons decays via a process with known or estimated branching fraction. There are a few modes that contribute in the $M_{\mathrm{bc}}$ signal region with a corresponding $\Delta E$ peak background. These peaking backgrounds are due to $K-\pi$ misidentification, including $B^{+} \rightarrow K^{+} K^{-} K^{+}, B^{+} \rightarrow K^{+} \pi^{-} \pi^{+}$, and their intermediate resonant modes.

The signal yield is extracted by performing a two-dimensional extended unbinned maximum likelihood fit in $M_{\mathrm{bc}}$ and $\Delta E$. Figure 6 shows the projection plots of the fit in the signal enhanced 
region. The resulting branching fraction is

$$
\mathscr{B}\left(B^{+} \rightarrow K^{+} K^{-} \pi^{+}\right)=(5.68 \pm 0.38 \pm 0.25) \times 10^{-6}
$$

and

$$
\mathscr{A}_{C P}=-0.177 \pm 0.067 \pm 0.006,
$$

where the quoted uncertainties are statistical and systematic, respectively.
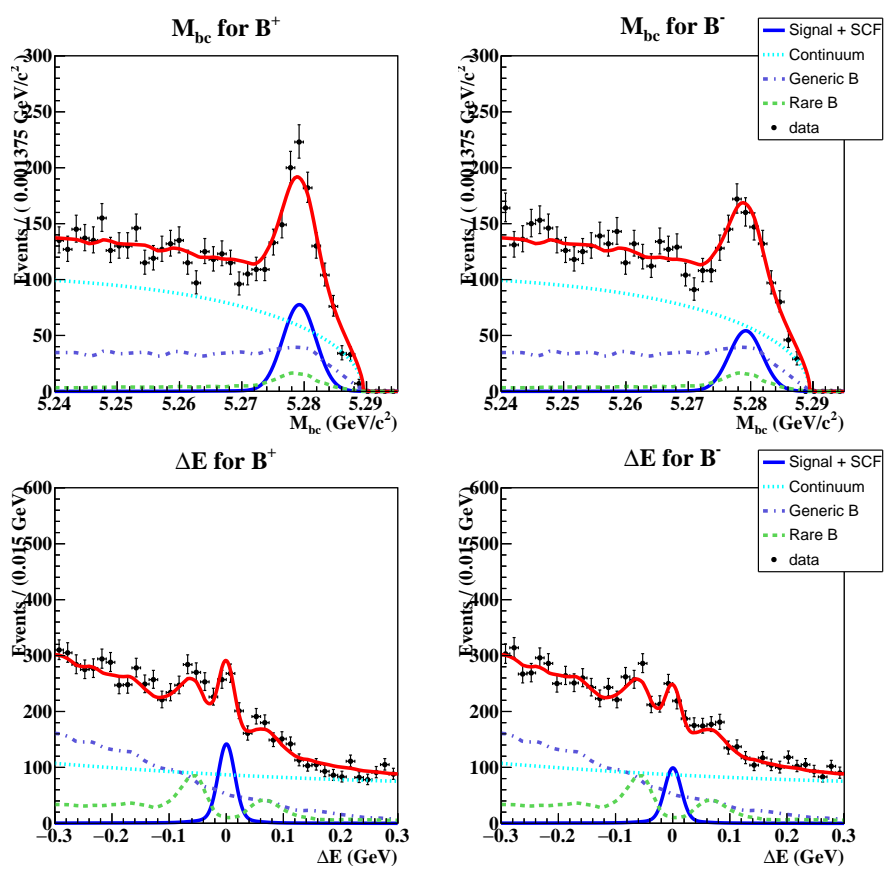

Figure 6: $M_{\mathrm{bc}}$ (top) and $\Delta E$ (bottom) distributions for the $B^{+}$(left) and $B^{-}$(right) candidates. Points with error bars are the data, the red line is the fit result, the blue line is the sum of the signal and the self cross-feed, the cyan dotted line is the continuum background, the purple dash-dot line is the generic $B$ backgrounds, and the green dashed line is the sum of rare $B$ backgrounds. The projection on $M_{\mathrm{bc}}$ is with the requirement of $-0.035<\Delta E<0.035 \mathrm{GeV}$, while the projection on $\Delta E$ is with the requirement of $5.275<M_{\mathrm{bc}}<5.285$ $\mathrm{GeV} / c^{2}$.

To investigate the localized $C P$ asymmetry in the low $M_{K^{+} K^{-}}$region, we determine the fitted signal yield and $\mathscr{A}_{C P}$ in bins of $M_{K^{+} K^{-}}$. The fitted results are shown in Table 2 and Fig. 7, where an excess and a large $\mathscr{A}_{C P}$ are seen in $M_{K^{+} K^{-}}<1.5 \mathrm{GeV} / c^{2}$, confirming the observations by BaBar and $\mathrm{LHCb}$. We find strong evidence of a large $C P$ asymmetry of $-0.90 \pm 0.17 \pm 0.03$ with $4.8 \sigma$ significance for $M_{K^{+} K^{-}}<1.1 \mathrm{GeV} / c^{2}$. 
Table 2: Signal yield and $\mathscr{A}_{C P}$ for individual $M_{K^{+} K^{-}}$bins. The first uncertainties are statistical and the second systematic. The signal yield is rescaled to the experimentally observed total $B^{+} \rightarrow K^{+} K^{-} \pi^{+}$signal yield.

\begin{tabular}{c|cc}
\hline \hline $\begin{array}{c}M_{K^{+} K^{-}} \\
\left(\mathrm{GeV} / c^{2}\right)\end{array}$ & $N_{\text {sig }}$ & $\mathscr{A}_{C P}$ \\
\hline $0.8-1.1$ & $63.9 \pm 12.2 \pm 3.6$ & $-0.90 \pm 0.17 \pm 0.03$ \\
$1.1-1.5$ & $227.0 \pm 22.8 \pm 10.7$ & $-0.16 \pm 0.10 \pm 0.01$ \\
$1.5-2.5$ & $121.3 \pm 28.6 \pm 19.7$ & $-0.15 \pm 0.23 \pm 0.03$ \\
$2.5-3.5$ & $117.7 \pm 18.8 \pm 6.1$ & $-0.09 \pm 0.16 \pm 0.01$ \\
$3.5-5.3$ & $184.6 \pm 27.5 \pm 9.9$ & $-0.05 \pm 0.15 \pm 0.00$ \\
\hline \hline
\end{tabular}
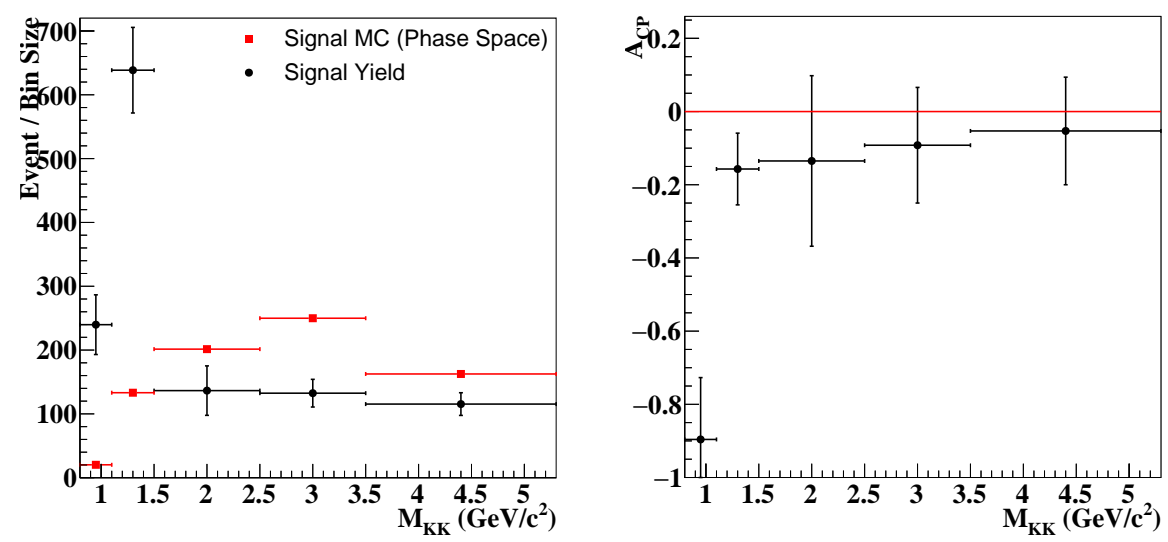

Figure 7: The fitted signal yield (left) and measured $\mathscr{A}_{C P}$ (right) as a function of $M_{K^{+} K^{-}}$. Each point is obtained from a two-dimensional fit with systematic uncertainty included. Red squares with error bars in the left figure show the expected signal distribution in a three-body phase space MC. Note that the phase space hypothesis is rescaled to the experimentally observed total $B^{+} \rightarrow K^{+} K^{-} \pi^{+}$signal yield.

\section{Belle II prospects for direct $C P$ violation in charmless $B$ decays}

Most of charmless $B$ decays are limited by the amount of data. The Belle II experiment aims to collect $50 \mathrm{ab}^{-1}$ data with high luminosity provided by SuperKEKB asymmetric-energy $e^{+} e^{-}$ collider. The high statistics enables us to study rare decays of $B$ mesons and to search for physics beyond the SM. The Belle II experiment is not just a renovation of the Belle experiment. Except the ECL crystals, all the sub-detectors are rebuilt. The VXD provides precise measurement of the primary and secondary vertices of short-lifetime particles, which helps to increase the reconstruction efficiency of $K_{S}^{0}$. The TOP detector improves the particle identification in the barrel region, while ARICH identifies particles in the forward endcap. The information from these two sub-detectors combined with that from the CDC improves the particle identification at Belle II, especially for $K / \pi$ separation. The Belle ECL is reused with improved back-end readout electronics with better waveform sampling, which improves the reconstruction efficiency of $\pi^{0}$ and $\gamma$. Figure 8 shows the 
Belle II detector.

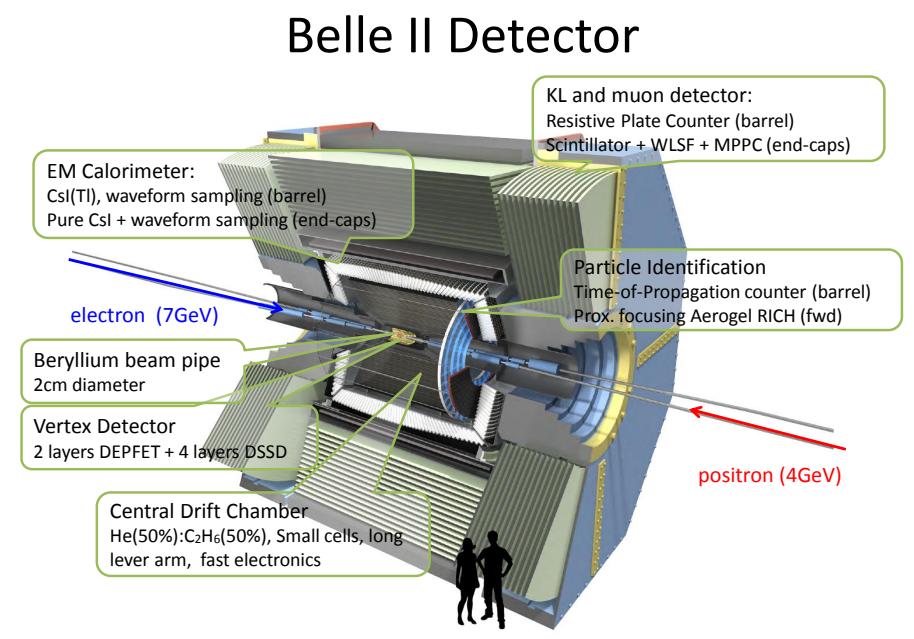

Figure 8: The Belle II detector.

With higher statistics, we will be able to study the direct $C P$ asymmetry in the charmless $B$ decays. The Belle II experiment will have over $1000 B_{s}^{0} \rightarrow K^{0} \bar{K}^{0}$ decays and over 30000 $B^{+} \rightarrow K^{+} K^{-} \pi^{+}$decays. Such a sample would allow for a much higher sensitivity search for new physics. With those improvements on particle identification and particle reconstruction, the uncertainty on measurements is also expected to be reduced. For example, in the $B \rightarrow K \pi$ system, the uncertainty on $\mathscr{A}_{C P}^{K^{0}} \pi^{0}$ is expected to reach $4 \%$, which helps to examine the sum rule for new physics [26].

\section{Conclusion}

We reported the recent results of charmless $B$ and $B_{s}^{0}$ decays using the full dataset collected by the Belle detector. Our measurement of branching fraction of $B_{s}^{0} \rightarrow K^{0} \bar{K}^{0}$ is the first observation of a charmless two-body decay in $B_{s}^{0}$ system involving only neutral hadrons. The first evidence of $B^{0} \rightarrow \eta \eta$ is presented which is consistent with the SM prediction. For the $B^{+} \rightarrow K^{+} K^{-} \pi^{+}$decays, we confirmed the observations by BaBar and LHCb and found a strong evidence of a large $C P$ asymmetry at the low $M_{K^{+} K^{-}}$region. Belle II will start operation in 2017 and start taking data with a complete detector in 2018 . With the high data statistics, we will be able to probe new physics in the charmless $B$ decays.

\section{References}

[1] Throughout this paper, the inclusion of the charge-conjugate mode is implied.

[2] C. H. Chen, "Analysis of $B_{s} \rightarrow K K$ decays in the PQCD," Phys. Lett. B 520, 33 (2001);

A. R. Williamson and J. Zupan, “Two body $B$ decays with isosinglet final states in SCET," Phys. Rev. D 74, 014003 (2006); A. Ali, G. Kramer, Y. Li, C. D. Lu, Y. L. Shen, W. Wang and Y. M. Wang, "Charmless non-leptonic $B_{s}$ decays to $P P, P V$ and $V V$ final states in the pQCD approach," Phys. Rev. 
D 76, 074018 (2007); C. K. Chua, "Rescattering effects in charmless $\bar{B}_{u, d, s} \rightarrow P P$ decays," Phys. Rev. D 78, 076002 (2008); K. Wang and G. Zhu, "Flavor dependence of annihilation parameters in QCD factorization,” Phys. Rev. D 88, 014043 (2013); J. J. Wang, D. T. Lin, W. Sun, Z. J. Ji, S. Cheng and Z. J. Xiao, “ $\bar{B}_{s}^{0} \rightarrow K \pi, K K$ decays and effects of the next-to-leading order contribution," Phys. Rev. D 89, 074046 (2014); Q. Chang, J. Sun, Y. Yang and X. Li, "A combined fit on the annihilation corrections in $B_{u, d, s} \rightarrow P P$ decays within QCDF," Phys. Lett. B 740, 56 (2015); H. Y. Cheng, C. W. Chiang and A. L. Kuo, "Updating $B \rightarrow P P, V P$ decays in the framework of flavor symmetry," Phys. Rev. D 91, 014011 (2015).

[3] S. Baek, D. London, J. Matias and J. Virto, " $B_{s}^{0} \rightarrow K^{+} K^{-}$and $B_{s}^{0} \rightarrow K^{0} \bar{K}^{0}$ decays within supersymmetry," J. High Energy Phys. 12, (2006) 019; A. Hayakawa, Y. Shimizu, M. Tanimoto and $\mathrm{K}$. Yamamoto, "Searching for the squark flavor mixing in $C P$ violations of $B_{s} \rightarrow K^{+} K^{-}$and $K^{0} \bar{K}^{0}$ decays," Prog. Theor. Exp. Phys. 2014, 023 B04 (2014).

[4] C.-C. Peng et al. (Belle Collaboration), "Search for $B_{s}^{0} \rightarrow h h$ decays at the $\Upsilon(5 S)$ resonance," Phys. Rev. D 82, 072007 (2010).

[5] Q. Chang, X. Q. Li and Y. D. Yang, "A comprehensive analysis of hadronic $b \rightarrow s$ transitions in a family non-universal $Z^{\prime}$ model,” J. Phys. G 41, 105002 (2014).

[6] S. Esen et al. (Belle Collaboration), "Precise measurement of the branching fractions for $B_{s} \rightarrow D_{s}^{(*)+} D_{s}^{(*)-}$ and first measurement of the $D_{s}^{*+} D_{s}^{*-}$ polarization using $e^{+} e^{-}$collisions," Phys. Rev. D 87, 031101(R) (2013).

[7] C. Oswald et al. (Belle Collaboration), "Semi-inclusive studies of semileptonic $B_{s}$ decays at Belle," Phys. Rev. D 92, 072013 (2015)

[8] K. A. Olive et al. (Particle Data Group), "Review of particle physics," Chin. Phys. C 38, 090001 (2014).

[9] B. Pal et al. (Belle Collaboration), Phys. Rev. Lett. 116, 161801 (2016).

[10] H.-Y. Chen and C.-K. Chua, Phys. Rev. D 80, 114008 (2009).

[11] A. R. Williamson and J. Zupan, Phys. Rev. D 74, 014003 (2006).

[12] C.-W. Chiang, M. Gronau, and J. L. Rosner, Phys. Rev. D 68, 074012 (2003).

[13] H.-K. Fu, X.-G. He, and Y.-K. Hsiao, Phys. Rev. D 69, 074002 (2004).

[14] B. Aubert et al. (BABAR Collaboration), Phys. Rev. D 79, 052003 (2009); Phys. Rev. Lett. 99, 161802 (2007); K.-F. Chen et al. (Belle Collaboration), Phys. Rev. Lett. 98, 031802 (2007).

[15] Y. Grossman and Z. Ligeti, Phys. Rev. D 68, 015004 (2003).

[16] M. Gronau, J. L. Rosner, and J. Zupan, Phys. Lett. B 596, 107 (2004).

[17] P. Chang et al. (Belle Collaboration), Phys. Rev. D 71, 091106 (2005).

[18] B. Aubert et al. (BABAR Collaboration), Phys. Rev. D 80, 112002 (2009).

[19] S. Kurokawa and E. and Kikutani, Nucl. Instrum. Meth. A 499, 1 (2003); T.Abe et al., Prog. Theor. Exp. Phys. 2013, 03A001 (2013).

[20] A. Abdesselam et al. (Belle Collaboration), arXiv:1609.03267 [hep-ex].

[21] B. Aubert et al. (BaBar Collaboration) Phys. Rev. Lett. 99, 221801 (2007).

[22] R. Aaij et al. (LHCb Collaboration) Phys. Rev. Lett. 112, 011801 (2014). 
[23] R. Aaij et al. (LHCb Collaboration) Phys. Rev. D 90, 112004 (2014).

[24] B. Bhattacharya, M. Gronau, and J. L. Rosner, Phys. Lett. B 726, 337 (2013).

[25] I. Bediaga, O. Lourenço, and T. Frederico, Phys. Rev. D 89, 094013 (2014).

[26] S. Baek et al., Phys. Lett. B 678, 97 (2009); M. Gronau, Phys. Lett. B 627, 82 (2005); D. Atwood, A. Soni, Phys. Rev. D 58, 036005 (1998). 